\title{
Luteolin induces myelodysplastic syndrome-derived cell apoptosis via the p53-dependent mitochondrial signaling pathway mediated by reactive oxygen species
}

\author{
WEIMIN DONG, YAN LIN, YANG CAO, YUE LIU, XIAOBAO XIE and WEIYING GU \\ Department of Hematology, The Third Affiliated Hospital of Soochow University, \\ The First People's Hospital of Changzhou, Changzhou, Jiangsu 213003, P.R. China
}

Received December 11, 2017; Accepted May 8, 2018

DOI: $10.3892 / \mathrm{ijmm} .2018 .3696$

\begin{abstract}
Luteolin, a common dietary flavonoid, induces the apoptosis of cells in several types of cancer. However, its role in myelodysplastic syndrome (MDS) and the potential underlying mechanisms remain to be elucidated. To evaluate the potential benefit and underlying mechanisms of luteolin in MDS cells, the viability of SKM-1 cells and primary bone marrow (PBM) mononuclear cells from patients with intermediate- or high-risk MDS were assessed using a Cell Counting Kit-8 assay. The apoptotic features of cell morphology were assessed using Wright-Giemsa staining, DNA fragmentation was analyzed by agarose gel electrophoresis, and the extent of apoptosis was quantified by flow cytometry (FCM). Reactive oxygen species (ROS) were measured by FCM with 2,7-dichlorodihydrofluorescein diacetate staining and mitochondrial membrane potential $(\Delta \Psi \mathrm{m})$ was determined using 5,5',6,6'-tetrachloro-1,1',3,3'-tetraethylbenzimidazolylcarbocyanine iodide staining. Caspase activity was detected using a fluorometric protease assay. Furthermore, the effects of luteolin on the expression of apoptosis-related proteins were analyzed using western blot analysis. The resulting data revealed that luteolin significantly inhibited the proliferation of SKM-1 cells in vitro, and its half maximal inhibitory concentration was
\end{abstract}

Correspondence to: Dr Weiying Gu, Department of Hematology, The Third Affiliated Hospital of Soochow University, The First People's Hospital of Changzhou, 185 Juqian Street, Tianning, Changzhou, Jiangsu 213003, P.R. China

E-mail: guweiying2001@163.com

Abbreviations: MDS, myelodysplastic syndrome; ROS, reactive oxygen species; $\Delta \Psi \mathrm{m}$, mitochondrial membrane potential; DMSO, dimethylsulfoxide; CCK8, Cell Counting Kit-8; FCM,flow cytometry; DCFH-DA, 2,7-dichlorodihydrofluorescein diacetate; JC-1, 5,5',6,6'-tetrachloro-1,1',3,3'-tetraethylbenzimidazolylcarbocyanine iodide; NAC, N-acetyl-L-cysteine; MFI, mean fluorescence intensity

Key words: luteolin, myelodysplastic syndrome, SKM-1 cells, reactive oxygen species, p53, mitochondrial membrane potential, apoptosis
$139.41 \mu \mathrm{M}$ at $24 \mathrm{~h}$ and $23.95 \mu \mathrm{M}$ at $72 \mathrm{~h}$. Luteolin also markedly inhibited the proliferation of mononuclear cells from patients with intermediate- or high-risk MDS. Luteolin suppressed cell proliferation, mainly as a result of the induction of apoptosis, as demonstrated by typical apoptotic morphological features, the ladder pattern of genomic DNA fragmentation, and the results of FCM using Annexin V-FITC/PI double staining. It was also found that short-term exposure of SKM-1 cells to luteolin led to a marked increase in the accumulation of ROS. The increased intracellular level of ROS appeared to induce the activation of p53 and elevate the B-cell lymphoma 2 (Bcl-2)-associated $\mathrm{X}$ protein $/ \mathrm{Bcl}-2$ ratio, which modulates $\Delta \Psi \mathrm{m}$ and triggers the release of cytochrome $c$, and may increase the activities of apoptotic protease activating factor 1 , caspase-3, -8 and -9 to further trigger the destruction of structural and specific proteins and thereby cell apoptosis. Notably, the inhibition of ROS generation by the antioxidant N-acetyl-L-cysteine significantly attenuated the luteolin-induced loss of $\Delta \Psi \mathrm{m}$ and activities of caspase-3, -8 and -9 . These data suggested that luteolin exerts its pro-apoptotic action partly through the p53-dependent mitochondrial signaling pathway mediated by intracellular ROS, which provides a promising therapeutic candidate for patients with MDS.

\section{Introduction}

Myelodysplastic syndromes (MDS) are a group of related clonal stem cell disorders characterized by ineffective hematopoiesis, peripheral blood cytopenia and an increased risk of progression to acute myeloid leukemia (1). Despite aggressive treatments, patients $>60$ years of age, or with secondary or therapy-related acute myeloid leukemia have the poorest prognosis, and those with high-risk MDS ineligible for allogeneic stem cell transplantation have similar outcomes, predominantly due to high rates of relapse (2). As previous studies have shown that only $5-15 \%$ of patients with therapy-related myelodysplasia or myeloid leukemia show long-term survival (3), the identification of novel effective agents with more benign toxicity profiles is required to improve the survival rate of patients with MDS.

Natural dietary compounds obtained from fruits, vegetables or herbs have been considered as potential chemopreventive 
and chemotherapeutic agents due to their non-toxic nature and usage for a long period of time without side effects (4). In preclinical studies, luteolin (3',4',5',7'-tetrahydroxyflavone), a type of flavonoid compound, has been reported to possess various pharmacological properties, including anti-inflammatory, antioxidant and anticancer activities (5-8), with anticancer activity having attracted increased attention in research (9). There is accumulating evidence that luteolin exerts its anticancer actions by affecting numerous biochemical pathways, which are critical for the regulation of cell proliferation, cell cycle arrest, apoptosis, angiogenesis, matrix metalloproteinase and metastasis (10-13). Additionally, luteolin has been demonstrated to inhibit the proliferation of leukemia cells and other cells through a mitochondrial-dependent pathway mediated by reactive oxygen species (ROS) $(9,14-17)$. Currently, the effect of luteolin on patients with MDS has not been addressed.

It is recognized that the induction of apoptosis of cancer cells is one of the most important and direct approaches to attenuate the development of cancer and eliminate tumors (18). Increasing evidence from multiple studies has shown that cell apoptosis is closely associated with the loss of mitochondrial inner membrane structure and integrity $(9,15)$. Mitochondria are central to the intrinsic apoptotic pathway, which involves a cascade of molecular events that occur entirely within the cell, and the increased ROS generation that occurs during apoptosis. It is known that ROS are a group of reactive, short-lived, oxygen-containing species, including superoxide, hydrogen peroxide, hydroxyl radicals, singlet oxygen, and peroxyl radicals (19). The overgeneration of ROS disrupts the balance between oxidation and antioxidant defense systems and, subsequently, it not only causes oxidative damage to proteins, lipids and nucleic acids, but also leads to the mitochondrial damage including collapse of the mitochondrial membrane potential $(\Delta \Psi \mathrm{m})$ and complex IV inactivation, resulting in mitochondrial dysfunction and consequent induction of cell apoptosis (20). Kittiratphatthana et al (17) reported that luteolin exerts its pro-apoptotic effect partly through generating intracellular ROS, which then contributes to the induction of mitochondria-mediated cell apoptosis. Others have indicated that the overexpression of ROS may contribute to the apoptosis through regulating B-cell lymphoma 2 (Bcl-2) family proteins, releasing cytochrome $c$ from the mitochondria, and activating effector caspases, including caspase-3 and caspase-9 (21). Therefore, a promising strategy for the prevention and treatment of MDS has emerged via inducing apoptosis by mitochondria-mediated ROS generation.

In the present study, SKM-1 cells and primary bone marrow (PBM) mononuclear cells from patients with intermediate- or high- risk MDS were used as models to evaluate the antiproliferative effect of luteolin on MDS cells. Furthermore, the possible molecular mechanism of luteolin-induced apoptosis in SKM-1 cells was examined, including the p53-dependent mitochondrial signaling pathway mediated by ROS and the expression of apoptosis-related proteins.

\section{Materials and methods}

Reagents and culture medium. Luteolin, obtained from Sigma; EMD Millipore (Billerica, MA, USA) was dissolved in dimethylsulfoxide (DMSO) and its concentration adjusted to $100 \mathrm{mM}$, as stock solutions, for storage at $-20^{\circ} \mathrm{C}$. This was diluted with medium prior to each experiment, in which the final concentration of DMSO was $<0.1 \%$. Fetal bovine serum (FBS) was obtained from Gibco; Thermo Fisher Scientific, Inc. (Waltham, MA, USA). Cell Counting Kit-8 (CCK8) was obtained from Beyotime Institute of Biotechnology (Haimen, China). Annexin V-FITC/PI double staining was obtained from BD Biosciences (Franklin Lakes, NJ, USA). The Fluorometric Protease Assay kit was obtained from Beyotime Institute of Biotechnology. 5,5',6,6'-tetrachloro-1,1',3,3'-tetraethylbenzimidazolylcarbocyanine iodide (JC-1) Mitochondrial Membrane Potential Assay Kit was obtained from Thermo Fisher Scientific, Inc. 2,7-dichlorodihydrofluorescein diacetate (DCFH-DA) and N-acetyl-L-cysteine (NAC) were obtained from Sigma-Aldrich; EMD Millipore. The BCA protein assay kit was obtained from Beijing Biosynthesis Biotechnology Co., Ltd. (Beijing, China). The anti-p53 (cat. no. 2527T), Bcl-2-associated X protein (Bax; cat. no. 5023T), Bcl-2 (cat. no. 2872T), apoptotic protease activating factor 1 (Apaf1; cat. no. 8969T), cytochrome $c$ (cat. no. 11940T), and $\beta$-actin (cat. no. 4967S) antibodies were obtained from Cell Signaling Technology, Inc. (Danvers, MA, USA). A secondary HRP-labeled goat anti-rabbit IgG (cat. no. ZB-2301) was obtained from Beijing Zhongshan Jinqiao Biotechnology Co., Ltd. (Beijing, China).

Cell culture. The SKM-1 cell line, originally established from a 76-year-old Japanese male patient with overt monoblastic leukemia following MDS (22), was obtained from the Institute of Biochemistry and Cell Biology, Shanghai Institutes for Biological Sciences, Chinese Academy of Sciences (Shanghai, China). The SKM-1 cells were grown in RPMI-1640 medium containing $10 \%$ heat-inactivated FBS, $100 \mathrm{U} / \mathrm{ml}$ penicillin and $100 \mu \mathrm{g} / \mathrm{ml}$ streptomycin in a humidified atmosphere of $5 \%$ $\mathrm{CO}_{2}$ at $37^{\circ} \mathrm{C}$, and the cultivating media was replaced every 2 days. SKM-1 cells in logarithmic growth phase were used in all experiments.

Primary cell culture. Between October 2017 and February 2018, PBM cells $\left(>1 \times 10^{6} / \mathrm{ml}\right)$ were obtained from the bone marrow of a patient with intermediate MDS (female, 71 years) and two patients with high-risk MDS (male, 75 years; and female, 52 years) in RPMI 1640 medium containing $100 \mathrm{U} / \mathrm{ml}$ heparin, following which the PBM mononuclear cells were isolated by gradient sedimentation using Ficoll-Hypaque $(1.077 \mathrm{~g} / \mathrm{ml})$. Subsequently, the cells were washed at least twice with PBS and cultured as described for the culture of SKM-1 cells above. All patients involved in the present study provided written informed consent and the Institutional Review Board of The Third Affiliated Hospital of Soochow University (Changzhou, China) approved the study.

Cytotoxicity assay. The cells were seeded in triplicate at a density of $5 \times 10^{3}$ cells/well (for SKM-1 cells) or $5 \times 10^{4}$ cells/well (for fresh MDS cells) in 96-well plates, respectively. Following incubation for $24 \mathrm{~h}$, serial dilutions of luteolin $(0,10,20,40,80$ and $120 \mu \mathrm{M}$ ) were added and incubated for various durations (12, 24 and 48 and $72 \mathrm{~h}$ ), following which $10 \mu \mathrm{l}$ of CCK8 solution $(5 \mathrm{~g} / \mathrm{l})$ was added to each well and the cells were incubated for another $3 \mathrm{~h}$ at $37^{\circ} \mathrm{C}$. Subsequently, the optical density for 
each well was measured using a Model 550 microplate reader (Bio-Rad Laboratories, Inc., Hercules, CA, USA) at a wavelength of $450 \mathrm{~nm}$ according to the manufacturer's protocol. Cell viability was calculated as the percentage of viable cells in the luteolin-treated group versus the control group. Finally, the obtained cell proliferation plots were used to calculate the half maximal inhibitory concentration $\left(\mathrm{IC}_{50}\right)$ value of the luteolin.

Analysis of apoptosis. To demonstrate the apoptotic features of the cells, the cells treated with or without luteolin were collected, sedimented onto microscopic slides and fixed with methanol. Wright-Giemsa staining (Yeasen Biotech, Shanghai, China) was then performed and images of the apoptotic morphological features were captured using an Olympus IX71 light microscope (Olympus Corporation, Tokyo, Japan). The extent of cell apoptosis was evaluated using Annexin V-FITC/PI double staining as previously reported (23).

DNA fragmentation assay. Following treatment with serial dilutions of luteolin for $24 \mathrm{~h}$, the SKM-1 cells were harvested and washed twice with sterile PBS. DNA was collected from the cells using TRIzol reagent according to manufacturer's protocol, and the quality and purity of the isolated DNA were then determined using a UV 2500 spectrophotometer (Shimadzu Corporation, Tokyo, Japan). The DNA samples $(15 \mu \mathrm{g})$ were then electrophoresed on a $1.5 \%$ agarose gel and visualized with ethidium bromide staining under ultraviolet light.

Measurement of ROS. Intracellular ROS levels were examined by flow cytometry (FCM) as described previously (24). Briefly, following incubation with serial dilutions of luteolin for $12 \mathrm{~h}$, DCFH-DA working solution was added directly to the culture medium at a final concentration of $10 \mu \mathrm{M}$. The cells were then incubated at $37^{\circ} \mathrm{C}$ for an additional $30 \mathrm{~min}$, following which the cells were collected and the intensity of fluorescence was analyzed by FACSCalibur FCM (BD Biosciences).

Determination of $\Delta \Psi m$. To demonstrate whether luteolin-induced apoptosis is mediated through mitochondrial dysfunction, alterations in $\Delta \Psi \mathrm{m}$ were detected by JC-1 staining. Briefly, the SKM-1 cells were pretreated with or without $5 \mathrm{mM}$ NAC for $2 \mathrm{~h}$ and then treated with serial dilutions of luteolin for $12 \mathrm{~h}$. The cells $\left(1 \times 10^{6}\right.$ cells $\left./ \mathrm{ml}\right)$ were suspended with PBS and incubated with JC-1 at a final concentration of $2 \mu \mathrm{M}$ for $20 \mathrm{~min}$ at $37^{\circ} \mathrm{C}$. The cells were then washed with $1 \mathrm{X}$ JC-1 buffer $(2 \mu \mathrm{M})$ and resuspended in PBS, and illuminated at $488 \mathrm{~nm}$. Images of $\Delta \Psi \mathrm{m}$ change from monomer and aggregate were captured using an FV-1000 fluorescence microscope (Olympus Corporation). Fluorescence-activated cell sorting analysis was performed by FCM, and the ratio of JC-1 monomers (green fluorescence) to JC-1 aggregates (orange fluorescence) was calculated for each concentration of luteolin and for the medium control.

Caspase activity assay. A Fluorometric Protease Assay kit was used to detect caspase activities according to the manufacturer's protocol. Briefly, the SKM-1 cells were preincubated with or without $5 \mathrm{mM} \mathrm{NAC}$ for $2 \mathrm{~h}$ and then incubated with serial dilutions of luteolin for $12 \mathrm{~h}$. The cells $\left(2 \times 10^{6}\right.$ cells $\left./ \mathrm{ml}\right)$ were collected and lysed by incubating them with lysis buffer provided in the kit on ice for $10 \mathrm{~min}$. Subsequently, $50 \mu \mathrm{g}$ of cell lysate proteins in $200 \mu \mathrm{l}$ of supernatant was incubated with an equal volume of reaction buffer containing fluorogenic peptide substrate at $37^{\circ} \mathrm{C}$ for $1 \mathrm{~h}$. The fluorescence intensities of cleaved substrate were then determined using a UV 2500 spectrophotometer (Shimadzu Corporation).

Western blot analysis. Cell protein extraction and western blot analysis were performed as described previously (25). Briefly, following treatment with luteolin, as described in the DNA fragmentation experiments, the total protein content of the cytosolic fraction in the supernatant was isolated from the harvested cells and measured using a BCA protein assay. The samples $(25 \mu \mathrm{g} /$ lane $)$ were subjected to $10 \%$ sodium dodecyl sulfate-polyacrylamide gel electrophoresis and transferred onto a nitrocellulose membrane. Nonspecific binding sites were blocked with $5 \%$ non-fat milk for $1 \mathrm{~h}$. The membranes were then incubated with primary antibodies against p53 (1:1,000), Bax (1:1,000), Bcl-2 (1:1,000), Apaf1 (1:1,000), cytochrome $c$ $(1: 1,000)$, and $\beta$-actin $(1: 1,000)$ for $2 \mathrm{~h}$ at $4^{\circ} \mathrm{C}$, and then with a secondary HRP-labeled goat anti-mouse IgG $(1: 2,000)$ for $1 \mathrm{~h}$ at room temperature. The blots were then visualized by enhanced chemiluminescence (ECL system, GE Healthcare Life Sciences, Chalfont, UK) and the density of $\beta$-actin served as an internal loading control.

Statistical analysis. Data are expressed as the mean \pm standard deviation and all analyses were performed with Statistical Package for Social Science (version 18.0; SPSS, Inc., Chicago, IL, USA). Comparisons between two groups were made using Student's t-test and those between three or more groups were made using one-way analysis of variance followed by the SNK test. $\mathrm{P}<0.05$ was considered to indicate a statistically significant difference.

\section{Results}

Inhibition of cell proliferation following treatment with luteolin. Following incubation with serial dilutions of luteolin for various durations, the CCK8 assay showed that the proliferation of SKM-1 cells was markedly inhibited in a time- and dose-dependent manner (Fig. 1A) and the dose of luteolin required to yield $\mathrm{IC}_{50}$ was $139.41 \mu \mathrm{M}$ at $24 \mathrm{~h}, 39.97 \mu \mathrm{M}$ at $48 \mathrm{~h}$, and $23.95 \mu \mathrm{M}$ at $72 \mathrm{~h}$. Notably, the cell viability of PBM mononuclear cells from one patient with intermediate-risk MDS was significantly decreased, compared with that in the medium control group $(\mathrm{P}<0.05)$ following treatment with 10 , 20, 40, 80 and $120 \mu \mathrm{M}$ luteolin for 24 and $48 \mathrm{~h}$. Similarly, the cell growth of primary cell cultures from another two patients with high-risk MDS was markedly inhibited, compared with that in the medium control group following treatment with the same concentration of luteolin for 24 and $48 \mathrm{~h}$ (all $\mathrm{P}<0.05$; Fig. 1B), although there were no significant differences in cell viability between patients. As luteolin exerted similar anticancer effects on the SKM-1 cells and PBM mononuclear cells from patients with MDS, SKM-1 cells were used as a model to examine the underlying mechanisms of luteolin in the subsequent experiments. 

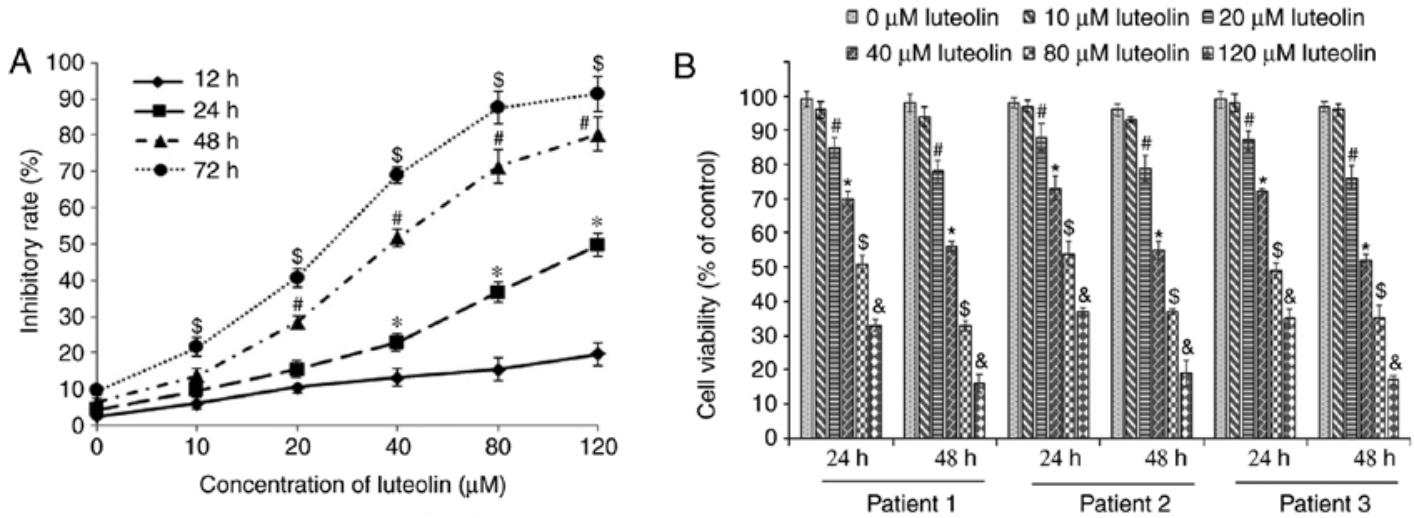

Figure 1. Effects of luteolin on MDS cell proliferation. (A) Inhibitory rate of SKM-1 cells. Following treatment with serial dilutions of luteolin for various durations, the optical density of SKM-1 cells was measured using a microplate reader. ${ }^{*} \mathrm{P}<0.05$ vs. cells treated with luteolin for $12 \mathrm{~h}$; ${ }^{*} \mathrm{P}<0.05 \mathrm{vs}$. cells treated with luteolin for $24 \mathrm{~h} ;{ }^{\$} \mathrm{P}<0.05$ vs. cells treated with luteolin for $48 \mathrm{~h}$. (B) Cell viability of PBM mononuclear cells. Following treatment with serial dilutions of luteolin for either 24 or $48 \mathrm{~h}$, the viability of PBM mononuclear cells from patients with intermediate-risk MDS (Patient 1) or high-risk MDS (Patients 2 and 3) were determined using a Cell Counting Kit- 8 assay. Data obtained from three independent experiments are presented as the mean \pm standard deviation. If error bars are not present, they are smaller than the thickness of the graph lines. ${ }^{\prime} \mathrm{P}<0.05$ vs. $10 \mu \mathrm{M}$ luteolin; ${ }^{*} \mathrm{P}<0.05$ vs. $20 \mu \mathrm{M}$ luteolin; ${ }^{\mathrm{S}} \mathrm{P}<0.05 \mathrm{vs} .40 \mu \mathrm{M}$ luteolin; ${ }^{\&} \mathrm{P}<0.05$ vs. $80 \mu \mathrm{M}$ luteolin. MDS, myelodysplastic syndrome; PBM, primary bone marrow.

Cell morphological assessment following treatment with luteolin. To determine whether the inhibition of cell growth by luteolin was in fact due to cell apoptosis, Wright-Giemsa staining was performed and the results demonstrated that the targeted cells underwent morphological changes characteristic of apoptosis. As expected, the typical morphological features of cell apoptosis, including cell shrinkage, chromatin condensation and loss of normal nuclear architecture, were observed in the luteolin-treated group under the light microscope, which were not observed in the control group (Fig. 2A and B).

DNA fragmentation analysis following treatment with luteolin. To confirm the induction of apoptosis by luteolin in SKM-1 cells, DNA was isolated and analyzed by agarose gel electrophoresis. As shown in Fig. 3A, a ladder-like pattern of DNA fragmentation became apparent in the SKM-1 cells following treatment with serial dilutions of luteolin for $24 \mathrm{~h}$, whereas no fragments were observed in the medium control group. In particular, a typical ladder pattern of genomic DNA fragmentation was observed in SKM-1 cells exposed to higher concentrations of luteolin $(40$ and $80 \mu \mathrm{M})$, in which the bands were thicker than those of $20 \mu \mathrm{M}$ luteolin, however, no significant difference was observed between the two groups treated with the two higher concentrations of luteolin (Fig. 3B).

Apoptotic induction of SKM-1 cells following treatment with luteolin. The effect of luteolin on the extent of cell apoptosis was further quantified by FCM. The apoptotic rates, including the sum of cells in early and late apoptosis, for the SKM-1 cells were increased in the luteolin-treated group, compared with those in the control group, and luteolin induced SKM-1 cell apoptosis in a dose-dependent manner (Fig. 4A-E), suggesting that $80 \mu \mathrm{M}$ luteolin had the largest pro-apoptotic effect on SKM-1 cells. In particular, the apoptotic rates of the SKM-1 cells were increased significantly with the prolonged duration of incubation with $40 \mu \mathrm{M}$ luteolin $(\mathrm{P}<0.05$; Fig. $4 \mathrm{~F})$.

Formation of intracellular ROS following treatment with luteolin. Following treatment with 0,20,40 and $80 \mu \mathrm{M}$ luteolin
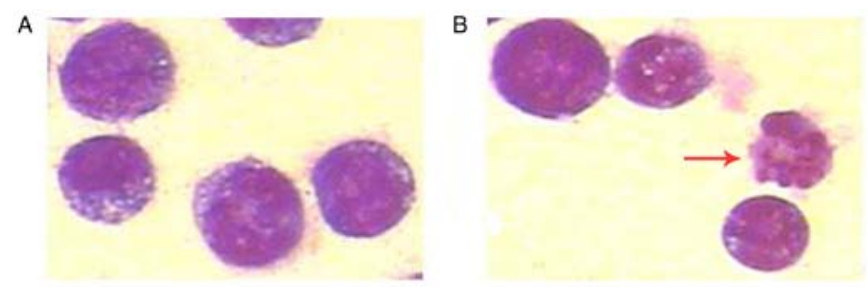

Figure 2. Morphological features of apoptosis in SKM-1 cells. (A) SKM-1 cells were treated with medium for $24 \mathrm{~h}$ as a control group. (B) SKM-1 cells were treated with $20 \mu \mathrm{M}$ luteolin for $24 \mathrm{~h}$, and observed under a light microscope. A representative image of three independent experiments is shown. The arrow indicates a typical apoptotic cell (Wright-Giemsa staining; magnification, $\mathrm{x} 1,000$ ).

for $12 \mathrm{~h}$, the mean fluorescence intensity (MFI) for the SKM-1 cells was increased, compared with that in the control group $(\mathrm{P}<0.05$; Fig. 5A). Compared with the corresponding time points for the serial dilutions of luteolin, a significant increase in MFI was observed in the $80 \mu \mathrm{M}$ luteolin group. In addition, following incubation with $40 \mu \mathrm{M}$ luteolin for different time intervals, the MFI of the SKM-1 cells was increased significantly with the prolonged duration of incubation $(\mathrm{P}<0.05$; Fig. 5B).

Depolarization of $\Delta \Psi m$ following treatment with luteolin. The cationic dye, JC-1, has the ability to enter into mitochondria and changes in color from green to orange reversibly. In normal cells with high $\Delta \Psi \mathrm{m}$, JC-1 spontaneously aggregates in mitochondria and emits red or orange fluorescence, and in those cells with low $\Delta \Psi \mathrm{m}$, JC-1 remains monomeric and emits green fluorescence. As shown in Fig. 6, the number of SKM-1 cells with orange fluorescence in the control group was higher (Fig. 6A) and the percentage of cells emitting only green fluorescence was increased following exposure of SKM-1 cells to $40 \mu \mathrm{M}$ luteolin (Fig. 6B). A shift from orange to green emission was observed under the fluorescence microscope, and there were 2.7- and 6.2-fold increases in loss of $\Delta \Psi \mathrm{m}$ in response to 20 and $40 \mu \mathrm{M}$ luteolin, respectively, compared with those in 

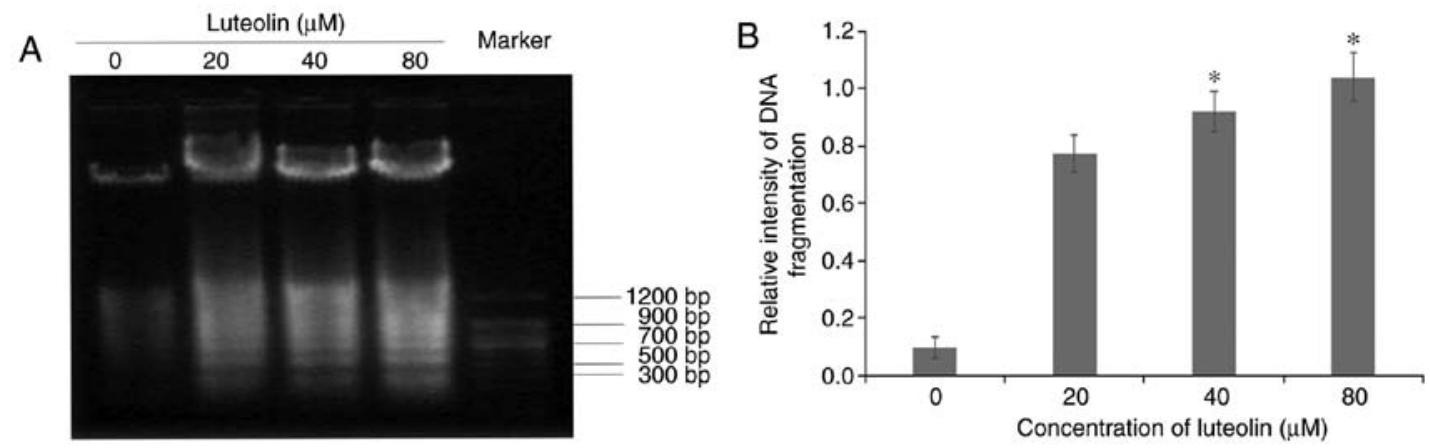

Figure 3. Effect of luteolin on DNA fragmentation in SKM-1 cells. (A) SKM-1 cells were treated with serial dilutions of luteolin for 24 h. DNA was then collected, electrophoresed on a 1.5\% agarose gel and visualized with ethidium bromide staining. A representative image of three independent experiments is shown. (B) Relative intensity of DNA fragmentation following treatment with serial dilutions of luteolin for $24 \mathrm{~h}$. Data obtained from three independent experiments are presented as the mean \pm standard deviation. ${ }^{*} \mathrm{P}<0.05$, vs. control group.
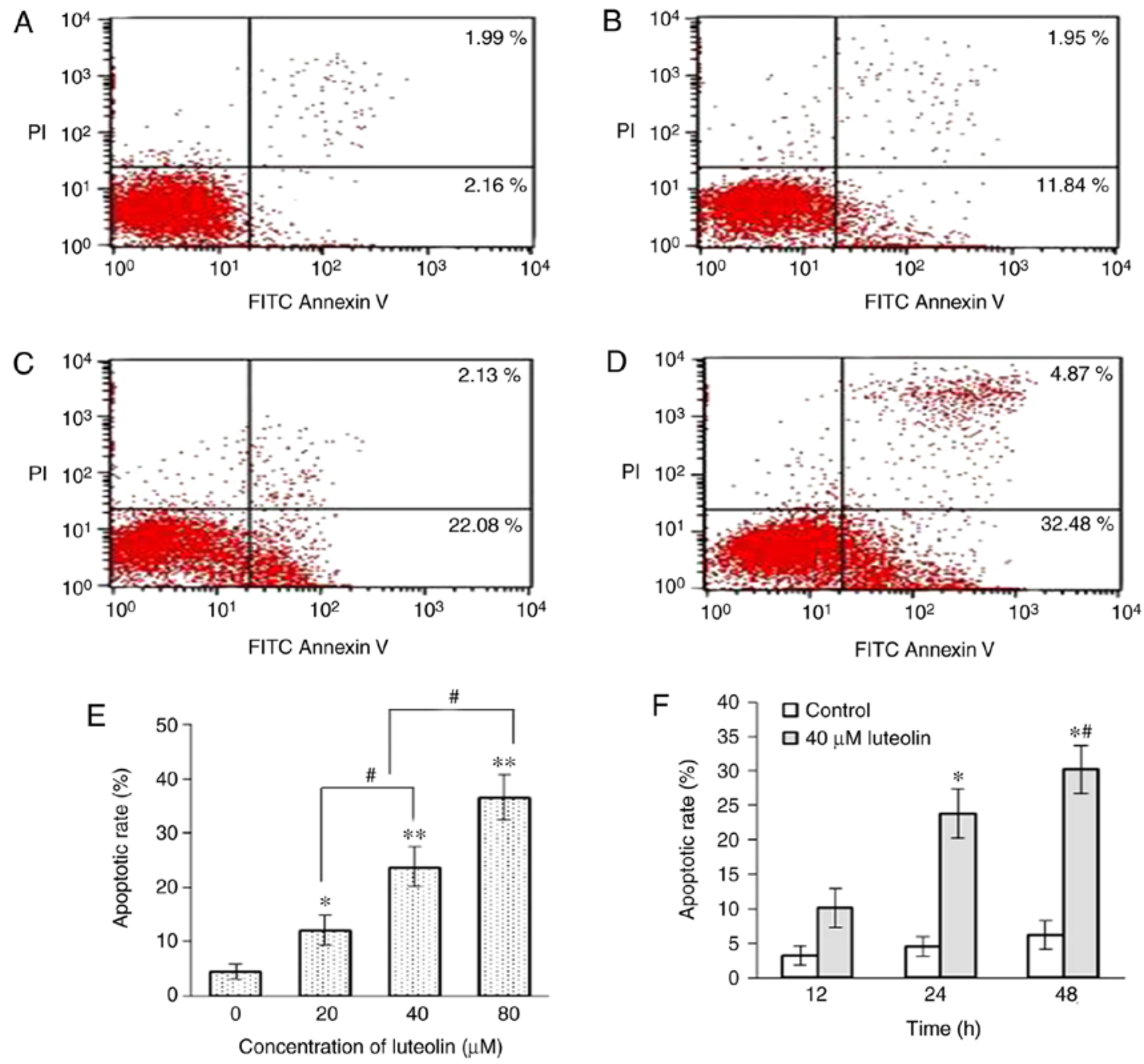

Figure 4. Effect of luteolin on the cell apoptosis for SKM-1 cells. SKM-1 cells were treated with (A) 0, (B) 20, (C) 40 and (D) $80 \mu$ M luteolin for 24 h, and evaluated using Annexin V-FITC/PI double staining. Results are representative of three independent experiments. Graph quadrants: Lower left, living cells (AV-negative/PI-negative); lower right, early apoptotic cells (AV-positive/PI-negative); upper right, late apoptotic cells (AV-positive/PI-positive); upper left, necrotic cells (AV-negative/PI-positive). The numbers of cells undergoing apoptosis represent the percentage of cells in the sum of the lower right and upper right quadrants. (E) Apoptotic rates of SKM-1 cells treated with serial dilutions of luteolin for $24 \mathrm{~h}$ were detected by FCM. Data obtained from three independent experiments are presented as the mean \pm standard deviation. ${ }^{*} \mathrm{P}<0.05$ and ${ }^{* *} \mathrm{P}<0.01$, vs. control group; ${ }^{*} \mathrm{P}<0.05$, vs. different concentration of luteolin. (F) Apoptotic rates of SKM-1 cells treated with $40 \mu \mathrm{M}$ luteolin for 12, 24 and $48 \mathrm{~h}$ were detected by FCM. Data obtained from three independent experiments are presented as the mean \pm standard deviation. ${ }^{*} \mathrm{P}<0.01$, vs. control group; ${ }^{*} \mathrm{P}<0.05$, vs. different time points with $40 \mu \mathrm{M}$ luteolin. FCM, flow cytometry.

the medium controls (Fig. 7). In particular, preincubation with the antioxidant NAC for $2 \mathrm{~h}$ effectively attenuated the loss of
$\Delta \Psi \mathrm{m}$ caused by luteolin, compared with that in the control group at the same time $(\mathrm{P}<0.05)$. 

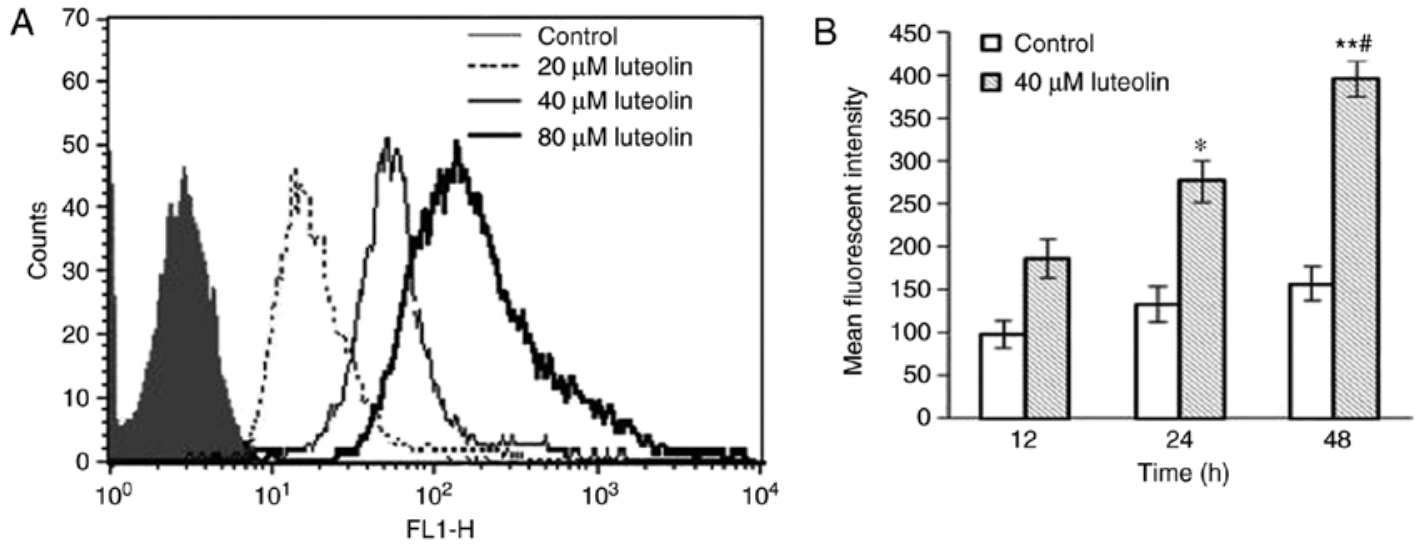

Figure 5. (A) Effect of luteolin on the accumulation of ROS in SKM-1 cells. SKM-1 cells were treated with serial dilutions of luteolin for $12 \mathrm{~h}$, stained with 2,7-dichlorodihydrofluorescein diacetate, and then quantified by flow cytometry. Images shown are representative profiles from three technical replicates. (B) Mean fluorescence intensity of SKM-1 cells following treatment with $40 \mu \mathrm{M}$ luteolin for 12, 24 and $48 \mathrm{~h}$. The data obtained from three independent experiments are presented as the mean \pm standard deviation. ${ }^{*} \mathrm{P}<0.05$ and ${ }^{* * *} \mathrm{P}<0.01$ vs. control group; ${ }^{~} \mathrm{P}<0.05$ vs. different time points with $40 \mu \mathrm{M}$ luteolin. ROS, reactive oxygen species.
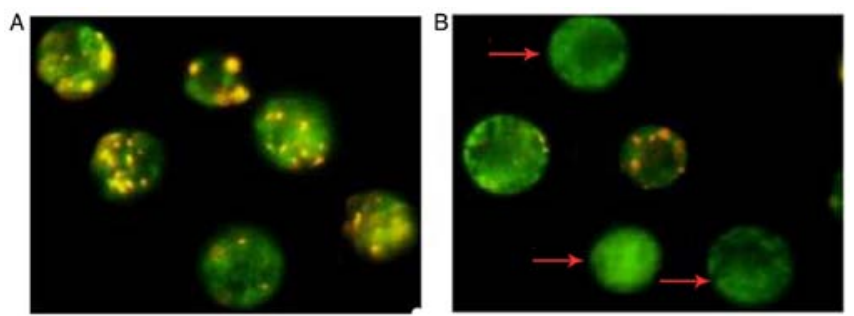

Figure 6. Typical fluorescence photomicrographs under a fluorescence microscope (JC-1 staining, magnification, x1,000). (A) SKM-1 cells were treated with medium for $12 \mathrm{~h}$ as a control group. (B) SKM-1 cells were treated with $40 \mu \mathrm{M}$ luteolin for $12 \mathrm{~h}$. Results shown are representative of two similar experiments. The orange fluorescence represents JC-1 aggregates, the green fluorescence represents JC-1 monomers, and the arrows indicate SKM-1 cells with a lower $\Delta \Psi \mathrm{m}$ in the luteolin-treated group compared with the control group. JC-1, 5,5',6,6'-tetrachloro-1,1',3,3'-tetraethylbenzimidazolylcarbocyanine iodide; $\Delta \Psi \mathrm{m}$, mitochondrial membrane potential.

Modulation of caspase activity following treatment with luteolin. Following incubation for $24 \mathrm{~h}$, luteolin markedly induced the activities of caspase-3, -8 and -9 in the SKM-1 cells, and there were significant differences compared with those in either the medium control group or NAC control group $(\mathrm{P}<0.05)$. The activities of caspases were higher in the NAC (-)/40 $\mu \mathrm{M}$ luteolin group, compared with those in the $\mathrm{NAC}(-) / 20 \mu \mathrm{M}$ luteolin group, suggesting that the induction of caspase activity occurred in a dose-dependent manner. Preincubation with the antioxidant NAC for $2 \mathrm{~h}$ effectively suppressed the activities of caspase-3, -8 and -9 induced by $40 \mu \mathrm{M}$ luteolin in the SKM-1 cells (Fig. 8).

Alteration of mitochondrial apoptosis related-proteins following treatment with luteolin. Following incubation with luteolin for $24 \mathrm{~h}$, the increased tumor suppressor protein $\mathrm{p} 53$, downregulation of anti-apoptotic protein $\mathrm{Bcl}-2$ and upregulation of proapoptotic proteins Bax, Apaf-1, and cytochrome $c$ in the SKM-1 cells were positively associated with the dose of luteolin (Fig. 9A) and there was a significant dose-dependent shift in the ratio of Bax to Bcl-2 (Fig. 9B).

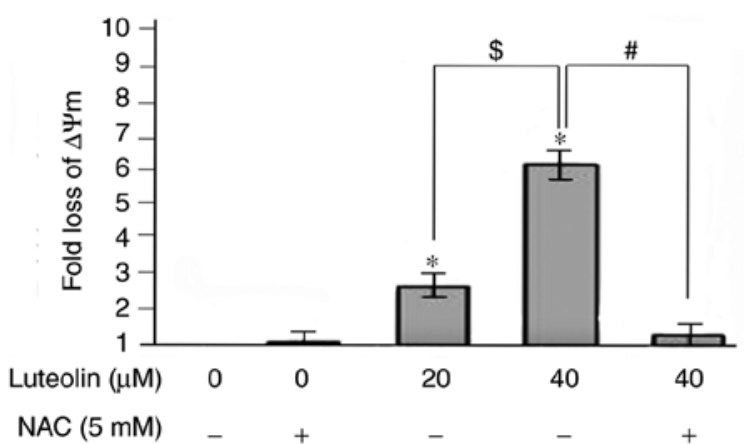

Figure 7. Fold loss of $\Delta \Psi \mathrm{m}$ in SKM-1 cells. SKM-1 cells were preincubated with or without $5 \mathrm{mM}$ NAC for $2 \mathrm{~h}$, and then incubated with serial dilutions of luteolin for $12 \mathrm{~h}$. The results derived from means of three independent experiments are presented as the mean \pm standard deviation. ${ }^{*} \mathrm{P}<0.05$, vs control group; ${ }^{\mathrm{P}} \mathrm{P}<0.01 \mathrm{NAC}(-) / 20 \mu \mathrm{M}$ luteolin group, vs. NAC(-)/40 $\mu \mathrm{M}$ luteolin group; ${ }^{*} \mathrm{P}<0.01 \mathrm{NAC}(-) / 40 \mu \mathrm{M}$ luteolin group, vs. $\mathrm{NAC}(+) / 40 \mu \mathrm{M}$ luteolin group. $\Delta \Psi \mathrm{m}$, mitochondrial membrane potential; NAC, N-acety-L-cystein.

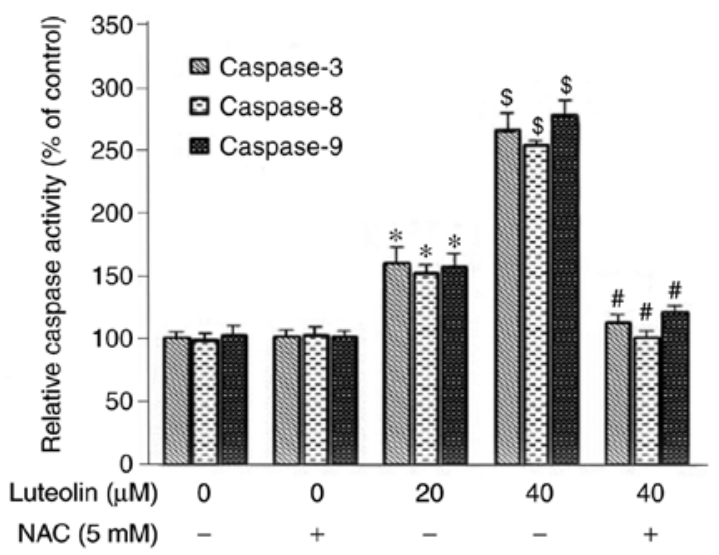

Figure 8. Relative caspase activity in SKM-1 cells. SKM-1 cells were preincubated with or without $5 \mathrm{mM}$ NAC for $2 \mathrm{~h}$, incubated with either 20 or $40 \mu \mathrm{M}$ luteolin for $12 \mathrm{~h}$, and then detected using a spectrophotometer. The data obtained from three independent experiments are presented as the mean \pm standard deviation. If error bars are not present, they are smaller than the thickness of graph lines. ${ }^{*} \mathrm{P}<0.05$, vs. control group or NAC control group; ${ }^{\$} \mathrm{P}<0.01$, vs. NAC(-)/20 $\mu \mathrm{M}$ luteolin group; ${ }^{\#} \mathrm{P}<0.01$, vs. NAC(-)/40 $\mu \mathrm{M}$ luteolin group. NAC, N-acety-L-cystein. 

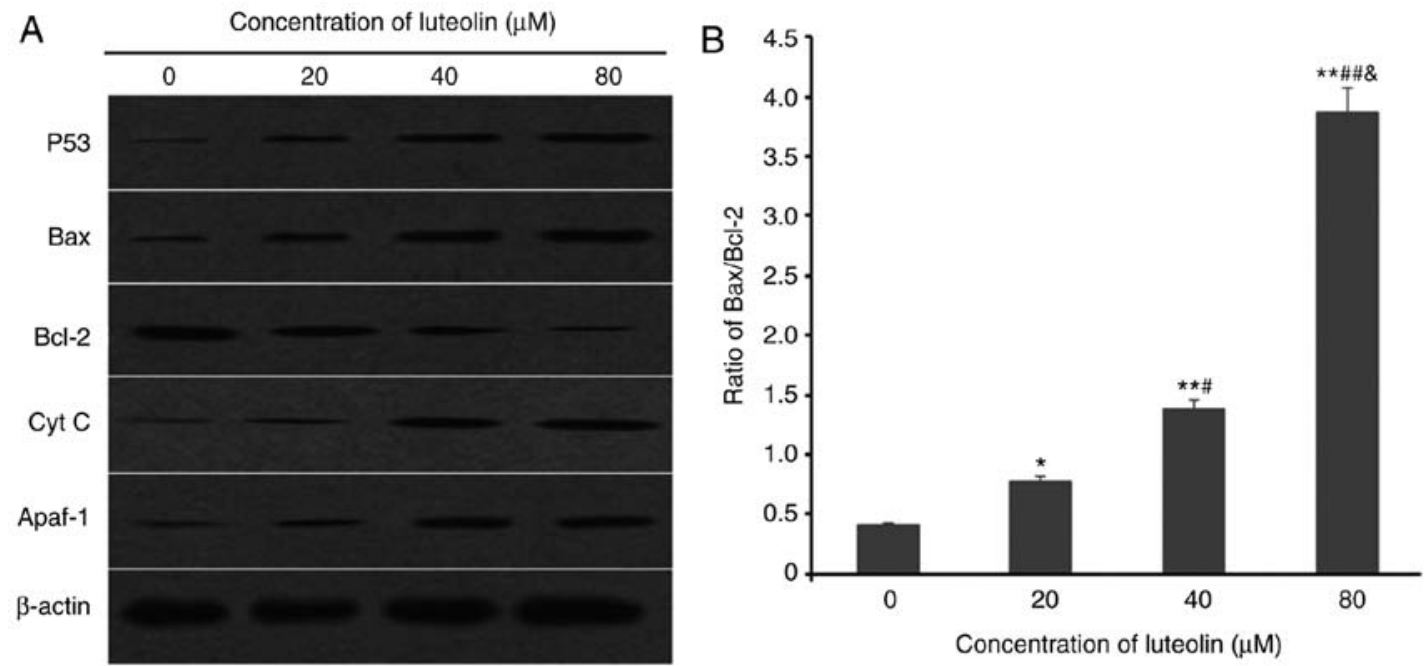

Figure 9. Effect of luteolin on the expression of mitochondrial apoptosis-related proteins. (A) SKM-1 cells were treated with serial dilutions of luteolin for $24 \mathrm{~h}$, and then examined using western blot analysis. Images shown are representative profiles from three technical replicates. (B) Ratio of Bax/Bcl-2 in SKM-1 cells following treatment with serial dilutions of luteolin for $24 \mathrm{~h}$. The data obtained from three independent experiments are presented as the mean \pm standard deviation. If error bars are not present, they are smaller than the thickness of graph lines. ${ }^{*} \mathrm{P}<0.05$ and ${ }^{* *} \mathrm{P}<0.01$ vs. control group; ${ }^{\#} \mathrm{P}<0.05$ and ${ }^{\# \#} \mathrm{P}<0.01$ vs. $20 \mu \mathrm{M}$ luteolin group; ${ }^{\&} \mathrm{P}<0.05$ vs. $40 \mu \mathrm{M}$ luteolin group. Bcl-2, B-cell lymphoma 2; Bax, Bcl-2-associated X protein; Cyt C, cytochrome $c$; Apaf1, apoptotic protease activating factor 1.

\section{Discussion}

Cytotoxic chemotherapy is used for patients with MDS with increasing myeloblasts and those who have progressed to acute leukemia. The incidence of MDS has been increasing rapidly with an aging population and an increasing number of individuals exposed to benzene (26). However, the unavailability of a curable approach and the blind strategy choice present challenges for the treatment of MDS. There has been increasing interest in the utilization of natural product-derived compounds as safe and effective chemotherapeutic agents for the treatment of various human diseases due to their diversity of biological activities. As a molecular targeted drug, the anticancer properties of luteolin have been identified to be associated with antiproliferative effects and pro-apoptotic effects, in addition to the inhibition of angiogenesis and metastasis $(9,14,27)$. However, the molecular mechanisms underlying its anticancer activities remain to be fully elucidated.

In the present study, the antiproliferative effect of luteolin on MDS cells was confirmed. The results of a CCK8 assay demonstrated that the proliferation of SKM-1 cells was markedly inhibited, and the dose of luteolin required to yield $\mathrm{IC}_{50}$ was $39.97 \mu \mathrm{M}$ at $48 \mathrm{~h}$, and $23.95 \mu \mathrm{M}$ at $72 \mathrm{~h}$. Luteolin also markedly inhibited the proliferation of PBM mononuclear cells from patients with intermediate- or high-risk MDS. Using differentiated enterocytes and the Caco-2 human colon carcinoma cell line, Abdel Hadi et al (28) demonstrated that luteolin exhibited cytotoxic activity towards human colon cancer cells with minimal or no effect on normal cells. These results suggested that luteolin may be a potential chemopreventive agent with an improved safety profile. In addition, the present study suggested that luteolin suppressed MDS cell proliferation, mainly as a result of the induction of apoptosis, as demonstrated by typical morphological features of cell apoptosis, including cell shrinkage, chromatin condensation and loss of normal nuclear architecture, under the microscope using Wright-Giemsa staining. The formation of DNA fragments of oligonucleosomal size (180-200 bp) is considered to be a hallmark of apoptotic cell death in several cell types (29). In the present study, the agarose gel electrophoresis showed a typical ladder pattern of genomic DNA fragmentation in the nuclei isolated from SKM-1 cells (Fig. 3). This observation led to the hypothesis that nucleosomal DNA fragmentation may be involved in luteolin-induced apoptosis of MDS cells. In addition, to evaluate the extent of cell apoptosis, Annexin V-FITC/PI double staining was performed; the results of FCM showed additional evidence for the occurrence of apoptosis induced by luteolin in a dose- and time-dependent manner, compared with that in the control group (Fig. 4). Consistent with these results, it has been reported that treatment with luteolin inhibits cell proliferation and induces cell apoptosis in other hematopoietic diseases (14,30-32). Collectively, the results presented in the present study and those of others provide clear evidence that luteolin exhibits cytotoxic effects on cancer cells through the induction of apoptosis, and a luteolin concentration of $80 \mu \mathrm{M}$ in the present study had the most marked pro-apoptotic effect on the SKM-1 cells.

Apoptosis, the key factor stimulating programmed cell death in cancer cells, has become one of the major mechanisms for the elimination of several types of cancer cell through different pathways, including the mitochondrial pathway (intrinsic pathway), endoplasmic reticulum pathway, or death receptor-medicated apoptotic pathway (extrinsic pathway) (33). These three signaling pathways communicate and interact with one another in physiological conditions, and are important in regulating cell dissolution (34). Accumulating evidence suggests that mitochondria are not only the major source of intracellular ROS, but they are also the major targets of their detrimental effects in mammalian cells $(15,35)$. Luteolin exerts its function as an antioxidant and ROS-generating agent (36), and various contrasting reports have been published regarding the effects of luteolin in the generation of ROS. In previous 
studies, Zhang et al (6) and Shelton et al (37) reported that luteolin possesses direct and indirect antioxidant effects by scavenging ROS. In agreement, Liu et al demonstrated that luteolin scavenges ROS generation to protect cells at concentrations of 1.0 and $10 \mu \mathrm{M}(38)$, whereas others $(16,19)$ reported that luteolin induces ROS accumulation in an early phase via the suppression of cellular superoxide dismutase activity in cancer cells. This discrepancy may be influenced by cell type and the dose of the ROS-inducing agents examined. In the present study, a significant increase in the MFI of SKM-1 cells was detected with the increase in luteolin concentration. Additionally, the MFI of the SKM-1 cells increased significantly with the prolonging of luteolin treatment duration, suggesting that luteolin leads to an intracellular accumulation of ROS in a dose-and time-dependent manner in MDS cells. It is well known that the excessive generation and accumulation of intracellular ROS can cause disruption of $\Delta \Psi \mathrm{m}$, which results in mitochondrial dysfunction and triggers cell apoptosis through multiple downstream signaling pathways (39). Similarly, Choi et al reported that a decrease in $\Delta \Psi \mathrm{m}$ following intense ROS generation induced mitochondrial disruption, inhibition of mitochondrial respiratory chain, reduction of ATP synthesis, and cell death (40). In the present study, the Annexin V-FITC/PI double staining and JC-1 staining showed that the dose of luteolin used in the experiments resulted in SKM-1 cell apoptosis, and this event was preceded by sustained hyperpolarization of the mitochondrial membrane, as demonstrated by the results of FCM and fluorescent microscope. A shift from orange to green emission was observed under the fluorescence microscope, and there was a higher fold increase in loss of $\Delta \Psi \mathrm{m}$ in response to the increase of luteolin concentration, compared with that in the control group. Preincubation with the antioxidant NAC effectively attenuated the loss of $\Delta \Psi \mathrm{m}$ caused by luteolin, compared with that in the control group at the same time. The results of these experiments indicated that luteolin inhibited cell proliferation and induced cell apoptosis through the mitochondrial pathway, mediated by ROS, in MDS cells.

P53, a tumor suppressor protein, is involved in inducing cell apoptosis (41). There is increasing evidence that luteolin activates the intrinsic apoptosis pathway through inducing DNA damage and activating the marked p53-dependent inhibition of cell proliferation and cell apoptosis in several cancer cell lines, including EC1 and KYSE450 esophageal carcinoma cells (9), HepG2 cells (42), cervical cancer cells (43), HT-29 colon cancer cells (44) and human RPE cells (45). Several lines of evidence have demonstrated that overexpression of the p53 gene suppresses the growth of human tumor cell lines $(46,47)$. The results of the present study confirmed that, following incubation with luteolin for $24 \mathrm{~h}$, the protein expression of $\mathrm{p} 53$ was augmented with the increasing concentration of luteolin. Therefore, these findings and those of others indicate that the $\mathrm{p} 53$ protein may act as a major activator for the induction of apoptosis in luteolin-treated SKM-1 cells. Until now, the mechanisms by which p53 regulates cell apoptosis have not been well illustrated, however, Bcl-2 family proteins, which comprise pro-apoptotic and anti-apoptotic members, are important in p53-dependent apoptosis (41). It has been shown that p53 directly activates the transcription of several genes encoding members of the Bcl-2 family, which can positively or negatively regulate mitochondrial outer membrane permeabilization to promote the release of cytochrome $c$ and other apoptotic molecules (48). To further examine the underlying mechanisms of p53-mediated apoptosis in luteolin-treated SKM-1 cells, the protein expression levels of Bax and Bcl-2 were determined by western blot analysis in the present study. The results confirmed that the mechanisms of luteolin-induced cell apoptosis may involve the downregulation of Bcl-2 and upregulation of Bax proteins in a dose-dependent manner, leading to a significant dose-dependent shift in the ratio of Bax to Bcl-2 family proteins in favor of the activation of caspase-3 in MDS cells. These findings are consistent with the findings of other studies that luteolin can activate the protein levels of Bax and Bcl-2 in human osteosarcoma, lung adenocarcinoma and gliomablastoma cells $(39,49,50)$. Collectively, these findings suggested that luteolin induces cell apoptosis via the p53-dependent mitochondrial signaling pathway by modulating a number of target proteins, particularly Bcl-2 and Bax.

It is known that the induction of apoptosis is accompanied by the release of cytochrome $c$ from mitochondria, which is considered one of the most important pro-apoptotic proteins and key in the assembly of a multimolecular complex known as the apoptosome. Its levels are markedly reduced in the normal cell cytoplasm and markedly increased in apoptotic cells (51). The increased generation of ROS induces the release of cytochrome $c$ from mitochondria to the cytoplasm (17), where it binds Apaf-1. The subsequent exchange of ADP/dADP for ATP/dATP in Apaf-1 triggers it to form an apoptosome (52). Therefore, the expression of cytochrome $c$ was analyzed in the present study to examine whether $c$ ytochrome $c$ had been released from the mitochondrial membrane in MDS cells, and to determine how cytochrome $c$ activates the downstream effector caspases to induce apoptosis in SKM-1 cells following incubation with luteolin. The results indicated that the levels of cytochrome $c$ increased significantly with the processing of caspase in a dose-dependent manner, compared with those in the control group. In addition, following incubation with luteolin for $24 \mathrm{~h}$, the accumulation of ROS appeared to upregulate the proapoptotic protein Apaf-1 in SKM-1 cells. Luteolin has been found to increase the expression of Bax and caspase- 3 and decrease the expression of Bcl-2 in liver carcinoma cells, but exerted almost no effects on normal liver HL-7702 cells (53). Therefore, it is reasonable to suggest that luteolin-induced ROS generation may be an early event in MDS cells, which promotes the induction of cell apoptosis by the release of cytochrome c from mitochondria.

Caspases are another important family of proteins involved in the downstream events of p53-mediated apoptosis (41). Caspase-3, an aspartic acid-specific cysteine protease, is the most widely studied mammalian caspase, which activates as cleaved caspase- 3 by specific shearing off the inactive subunit in the location of aspartic acid. Additionally, it is important in both death pathways, and cleaves a wide range of cellular substrates (54). To elucidate whether caspase activation was involved in luteolin-induced apoptosis, a fluorometric assay was performed to detect the activities of caspase-3, -8 , and -9 . Following incubation for $24 \mathrm{~h}$, luteolin significantly induced the activation of caspase- $3,-8$ and -9 in SKM-1 cells in a 
dose-dependent manner. Preincubation with the antioxidant NAC for $2 \mathrm{~h}$ effectively suppressed the activity of caspase-3, -8 and -9 induced by $40 \mu \mathrm{M}$ luteolin (Fig. 8). These results supported the hypothesis that luteolin induces ROS-mediated apoptosis by activating caspase-dependent pathways.

In conclusion, luteolin was shown to inhibit the proliferation of SKM-1 cells and exerted its pro-apoptotic action partly through the $\mathrm{p} 53$-dependent mitochondrial signaling pathway mediated by intracellular ROS. This provides a promising therapeutic candidate for patients with MDS. However, further investigation of luteolin in clinical trials is required to confirm these results.

\section{Acknowledgements}

The authors would like to thank Dr Feng Gao for technical assistance.

\section{Funding}

This study was supported by the Fund for Young Scholars in Jiangsu Province (grant no. BK 20150253), the Fund of Science and Technology Bureau of Changzhou, Jiangsu, China (grant no. CJ20150418) and the Changzhou High-Level Medical Talents Training Project (grant no. 2016ZCLJ024).

\section{Availability of data and materials}

All datasets generated and analysed in the present study are included in this published article.

\section{Authors' contributions}

WD and WG conceived and designed the study. YaL, YC and YuL performed all the experiments. XX performed all the clinical diagnoses and treatments. YaL and YC analyzed the data. WD and YaL wrote the manuscript. WG revised the manuscript. All authors have read and approved the final manuscript.

\section{Ethics approval and consent to participate}

All patients involved in the present study provided the written informed consent and the Institutional Review Board of The Third Affiliated Hospital of Soochow University approved the study.

\section{Consent for publication}

All the study participants provided consent for the data to be published.

\section{Competing interests}

The authors declare that they have no competing interests.

\section{References}

1. Sekeres MA and Cutler C: How we treat higher-risk myelodysplastic syndromes. Blood 123: 829-836, 2014.
2. Ferrero D, Crisà E, Marmont F, Audisio E, Frairia C, Giai V, Gatti T, Festuccia M, Bruno B, Riera L, et al: Survival improvement of poor-prognosis AML/MDS patients by maintenance treatment with low-dose chemotherapy and differentiating agents. Ann Hematol 93: 1391-1400, 2014.

3. Smith SM, Le Beau MM, Huo D, Karrison T, Sobecks RM, Anastasi J, Vardiman JW, Rowley JD and Larson RA: Clinical-cytogenetic associations in 306 patients with therapy-related myelodysplasia and myeloid leukemia: The University of Chicago series. Blood 102: 43-52, 2003.

4. Khan N, Afaq F, Syed DN and Mukhtar H: Fisetin, a novel dietary flavonoid, causes apoptosis and cell cycle arrest in human prostate cancer LNCaP cells. Carcinogenesis 29: 1049-1056, 2008.

5. Wang SX, Cao M, Xu SH, Zhang JM, Wang ZG, Mao XD, Yao XM and Liu C: Effect of luteolin on inflammatory responses in RAW264.7 macrophages activated with LPS and IFN- $\gamma$. J Funct Foods 32: 123-130, 2017.

6. Zhang YC, Gan FF, Shelar SB, Ng KY and Chew EH: Antioxidant and Nrf2 inducing activities of luteolin, a flavonoid constituent in Ixeris sonchifolia Hance, provide neuroprotective effects against ischemia-induced cellular injury. Food Chem Toxicol 59: 272-280, 2013.

7. Lu J, Li G, He K, Jiang W, Xu C Li Z, Wang H, Wang W, Wang H, Teng $X$ and Teng L: Luteolin exerts a marked antitumor effect in cMet-overexpressing patient-derived tumor xenograft models of gastric cancer. J Transl Med 13: 42, 2015.

8. Suh KS, Chon S and Choi EM: Luteolin alleviates methylglyoxal-induced cytotoxicity in osteoblastic MC3T3-E1 cells. Cytotechnology 68: 2539-2552, 2016.

9. Chen P, Zhang JY, Sha BB, Ma YE, Hu T, Ma YC, Sun H, Shi JX, Dong ZM and Li P: Luteolin inhibits cell proliferation and induces cell apoptosis via down-regulation of mitochondrial membrane potential in esophageal carcinoma cells EC1 and KYSE450. Oncotarget 8: 27471-27480, 2017.

10. Lim W, Yang C, Bazer FW and Song G: Luteolin inhibits proliferation and induces apoptosis of human placental choriocarcinoma cells by blocking the PI3K/AKT pathway and regulating sterol regulatory element binding protein activity. Biol Reprod 95: 82, 2016.

11. Park SH, Ham S, Kwon TH, Kim MS, Lee DH, Kang JW, Oh SR and Yoon DY: Luteolin induces cell cycle arrest and apoptosis through extrinsic and intrinsic signaling pathways in MCF-7 breast cancer cells. J Environ Pathol Toxicol Oncol 33: 219-231, 2014.

12. Pandurangan AK, Dharmalingam P, Sadagopan SK and Ganapasam S: Luteolin inhibits matrix metalloproteinase 9 and 2 in azoxymethane-induced colon carcinogenesis. Hum Exp Toxicol 33: 1176-1185, 2014.

13. Tsai PH, Cheng CH, Lin CY, Huang YT, Lee LT, Kandaswami CC, Lin YC, Lee KP, Hung CC, Hwang JJ, et al: Dietary flavonoids luteolin and quercetin suppressed cancer stem cell properties and metastatic potential of isolated prostate cancer cells. Anticancer Res 36: 6367-6380, 2016.

14. Deng L, Jiang L, Lin X, Tseng KF, Lu Z and Wang X: Luteolin, a novel p90 ribosomal S6 kinase inhibitor, suppresses proliferation and migration in leukemia cells. Oncol Lett 13: 1370-1378, 2017.

15. Chen HM, Tang XX, Zhou B, Zhou Z, Xu N and Wang Y: A ROS-mediated mitochondrial pathway and Nrf2 pathway activation are involved in BDE-47 induced apoptosis in Neuro-2a cells. Chemosphere 184: 679-686, 2017.

16. Wang W, Zhao FI, Zhang J and Gao D: Luteolin induces apoptosis in mouse liver cancer cells through ROS mediated pathway: A mechanistic investigation. Biomed Res 28: 839-845, 2017.

17. Kittiratphatthana N, Kukongviriyapan V, Prawan A and Senggunprai L: Luteolin induces cholangiocarcinoma cell apoptosis through the mitochondrial-dependent pathway mediated by reactive oxygen species. J Pharm Pharmacol 68: 1184-1192, 2016.

18. Gerl R and Vaux DL: Apoptosis in the development and treatment of cancer. Carcinogenesis 26: 263-270, 2005.

19. Ju W, Wang X, Shi H, Chen W, Belinsky SA and Lin Y: A critical role of luteolin-induced reactive oxygen species in blockage of tumor necrosis factor-activated nuclear factor-kappa B pathway and sensitization of apoptosis in lung cancer cells. Mol Pharmacol 71: 1381-1388, 2007.

20. Ding GL, Zhao JQ and Jiang DM: Allicin inhibits oxidative stress-induced mitochondrial dysfunction and apoptosis by promoting PI3K/AKT and CREB/ERK signaling in osteoblast cells. Exp Ther Med 11: 2553-2560, 2016. 
21. Liu BX, Zhou JY, Li Y, Zou X, Wu J, Gu JF, Yuan JR, Zhao BJ, Feng L, Jia XB and Wang RP: Hederagenin from the leaves of ivy (Hedera helix L.) induces apoptosis in human LoVo colon cells through the mitochondrial pathway. BMC Complement Altern Med 14: 412, 2014.

22. Nakagawa T, Matozaki S, Murayama T, Nishimura R, Tsutsumi M, Kawaguchi R, Yokoyama Y, Hikiji K, Isobe T and Chihara K: Establishment of a leukaemic cell line from a patient with acquisition of chromosomal abnormalities during disease progression in myelodysplastic syndrome. Br J Haematol 85: 469-761, 1993.

23. Xia G, Chen B, Ding J, Gao C, Lu H, Shao Z, Gao F and Wang X: Effect of magnetic $\mathrm{Fe}_{3} \mathrm{O}_{4}$ nanoparticles with 2-methoxyestradiol on the cell-cycle progression and apoptosis of myelodysplastic syndrome cells. Int J Nanomedicine 6: 1921-1927, 2011.

24. Kim SY, Lee YM and Cho JS: Korean red ginseng extract exhibits neuroprotective effects through inhibition of apoptotic cell death. Biol Pharm Bull 37: 938-946, 2014.

25. Lu H, Gao F, Shu G, Xia G, Shao Z, Lu H and Cheng K: Wogonin inhibits the proliferation of myelodysplastic syndrome cells through the induction of cell cycle arrest and apoptosis. Mol Med Rep 12: 7285-7292, 2015.

26. Jing Y, Shen X, Mei Q and Han W: Spotlight on decitabine for myelodysplastic syndromes in Chinese patients. Onco Targets Ther 8: 2783-2790, 2015

27. Kim YS, Kim SH, Shin J, Harikishore A, Lim JK, Jung Y, Lyu HN, Baek NI, Choi KY, Yoon HS and Kim KT: Luteolin suppresses cancer cell proliferation by targeting vaccinia-related kinase 1. PLoS One 9: e109655, 2014.

28. Abdel Hadi L, Di Vito C, Marfia G, Ferraretto A, Tringali C, Viani $\mathrm{P}$ and Riboni L: Sphingosine kinase 2 and ceramide transport as key targets of the natural flavonoid luteolin to induce apoptosis in colon cancer cells. PloS One 10: e0143384, 2015.

29. Devi PS, Kumar MS and Das SM: Evaluation of antiproliferative activity of red sorghum bran anthocyanin on a human breast cancer cell line (mcf-7). Int J Breast Cancer 2011: 891481, 2011.

30. Cheng AC, Huang TC, Lai CS and Pan MH: Induction of apoptosis by luteolin through cleavage of Bcl-2 family in human leukemia HL-60 cells. Eur J Pharmacol 509: 1-10, 2005.

31. Ko WG, Kang TH, Lee SJ, Kim YC and Lee BH: Effects of luteolin on the inhibition of proliferation and induction of apoptosis in human myeloid leukaemia cells. Phytother Res 16: 295-298, 2002.

32. Sak K, Kasemaa K and Everaus H: Potentiation of luteolin cytotoxicity by flavonols fisetin and quercetin in human chronic lymphocytic leukemia cell lines. Food Funct 7: 3815-3824, 2016.

33. Fiandalo MV and Kyprianou N: Caspase control: Protagonists of cancer cell apoptosis. Exp Oncol 34: 165-175, 2012.

34. Schwartz JT, Barker JH, Kaufman J, Fayram DC, McCracken JM and Allen LA: Francisella tularensis inhibits the intrinsic and extrinsic pathways to delay constitutive apoptosis and prolong human neutrophil lifespan. J Immunol 188: 3351-3363, 2012.

35. Marchi S, Giorgi C, Suski JM, Agnoletto C, Bononi A, Bonora M, De Marchi E, Missiroli S, Patergnani S, Poletti F, et al: Mitochondria-ros crosstalk in the control of cell death and aging. J Signal Transduct 2012: 329635, 2012.

36. Rao PS, Satelli A, Moridani M, Jenkins M and Rao US: Luteolin induces apoptosis in multidrug resistant cancer cells without affecting the drug transporter function: Involvement of cell line-specific apoptotic mechanisms. Int J Cancer 130: 2703-2714 2012.

37. Shelton LM, Park BK and Copple IM: Role of Nrf2 in protection against acute kidney injury. Kidney Int 84: 1090-1095, 2013.
38. Liu R, Meng F, Zhang L, Liu A, Qin H, Lan X, Li L and Du G: Luteolin isolated from the medicinal plant Elsholtzia rugulosa (Labiatae) prevents copper-mediated toxicity in $\beta$-amyloid precursor protein Swedish mutation overexpressing SH-SY5Y cells. Molecules 16: 2084-2096, 2011.

39. Wang Q, Wang H, Jia Y, Pan H and Ding H: Luteolin induces apoptosis by ROS/ER stress and mitochondrial dysfunction in gliomablastoma. Cancer Chemother Pharmacol 79: 1031-1041, 2017.

40. Choi IY, Lee SJ, Ju C, Nam W, Kim HC, Ko KH and Kim WK: Protection by a manganese porphyrin of endogenous peroxynitrite-induced death of glial cells via inhibition of mitochondrial transmembrane potential decrease. Glia 31: 155-164, 2000.

41. Lin Y, Xu JP, Liao HH, Li L and Pan L: Piperine induces apoptosis of lung cancer A549 cells via p53-dependent mitochondrial signaling pathway. Tumor Biol 35: 3305-3310, 2014

42. Yee SB, Choi HJ, Chung SW, Park DH, Sung B, Chung HY and Kim ND: Growth inhibition of luteolin on HepG2 cells is induced via $\mathrm{p} 53$ and Fas/Fas-ligand besides the TGF- $\beta$ pathway. Int J Oncol 47: 747-754, 2015.

43. Ham S, Kim KH, Kwon TH, Bak Y, Lee DH, Song YS, Park SH, Park YS, Kim MS, Kang JW, et al: Luteolin induces intrinsic apoptosis via inhibition of E6/E7 oncogenes and activation of extrinsic and intrinsic signaling pathways in HPV-18-associated cells. Oncol Rep 31: 2683-2691, 2014.

44. Lim DY, Jeong Y, Tyner AL and Park JH: Induction of cell cycle arrest and apoptosis in HT-29 human colon cancer cells by the dietary compound luteolin. Am J Physiol Gastrointest Liver Physiol 292: G66-G75, 2007.

45. Hytti M, Szabó D, Piippo N, Korhonen E, Honkakoski P, Kaarniranta K, Petrovski G and Kauppinen A: Two dietary polyphenols, fisetin and luteolin, reduce inflammation but augment DNA damage-induced toxicity in human RPE cells. J Nutr Biochem 42: 37-42, 2017.

46. Ge Q, Wang C, Ruan Y, Chen Z, Liu J and Ye Z: Overexpression of p53 activated by small activating RNA suppresses the growth of human prostate cancer cells. Onco Targets Ther 9: 231-241, 2016.

47. Wang C, Ge Q, Zhang Q, Chen Z, Hu J, Li F and Ye Z: Targeted p53 activation by saRNA suppresses human bladder cancer cells growth and metastasis. J Exp Clin Cancer Res 35: 53, 2016

48. Macip S, Igarashi M, Berggren P, Yu J, Lee SW and Aaronson SA: Influence of induced reactive oxygen species in p53-mediated cell fate decisions. Mol Cell Biol 23: 8576-8585, 2003.

49. Wang YH, Kong DL, Wang XW, Dong X, Tao Y and Gong H: Molecular mechanisms of luteolin induced growth inhibition and apoptosis of human osteosarcoma cells. Iran J Pharm Res 14: 531-538, 2015.

50. Chen Q, Liu S, Chen J, Zhang Q, Lin S, Chen Z and Jiang J: Luteolin induces mitochondria-dependent apoptosis in human lung adenocarcinoma cell. Nat Prod Commun 7: 29-32, 2012.

51. Kilbride SM and Prehn JH: Central roles of apoptotic proteins in mitochondrial function. Oncogene 32: 2703-2711, 2013

52. Bao Q, Lu W, Rabinowitz JD and Shi Y: Calcium blocks formation of apoptosome by preventing nucleotide exchange in Apaf-1. Mol Cell 25: 181-192, 2007.

53. Ding S, Hu A, Hu Y, Ma J, Weng P and Dai J: Anti-hepatoma cells function of luteolin through inducing apoptosis and cell cycle arrest. Tumour Biol 35: 3053-3060, 2014.

54. Kobayashi T, Masumoto J, Tada T, Nomiyama T, Hongo K and Nakayama J: Prognostic significance of the immunohistochemical staining of cleaved caspase-3, an activated form of caspase-3, in gliomas. Clin Cancer Res 13: 3868-3874, 2007. 